\title{
ВЛИЯНИЕ ПОДЗАДАЧИ О РАСКРОЕ ПРИ ОЦЕНКЕ ЦЕЛЕСООБРАЗНОСТИ ПРОВЕДЕНИЯ ЗАКУПОК ЛЕСНОГО СЫРЬЯ С ТОВАРНО-СЫРЬЕВЫХ БИРЖ
}

\author{
(c) 2021 Р. С. Рогулин \\ Владивостокский государственный университет экономики и сервиса \\ ул. Гоголя 41, 690014 Владивосток, Российская Федерация
}

\begin{abstract}
Аннотация. В статье рассмотрена математическая модель, позволяющая найти оптимальный план закупок сырья с биржи по заранее известному распределению заявок во времени, определить план производства, вычислить оптимальный план доставки продукции, отличающуюся возможностью сформировать план раскроя плит ОСП на заготовки различных длин, а также наличием связи между ценой и объемом спроса на конечные товары. Целью работы является получение оценки экономической целесообразности взаимодействия предприятия лесопромышленной отрасли (при разных уровнях цен и спроса) и международной товарно-сырьевой биржи. Гипотеза заключается в том, что взаимодействие лесопромышленного комплекса с биржей может быть выгодным для предприятия. Актуальность исследования заключается в слабой проработке современной литературе вопроса взаимодействия лесопромышленного предприятия с биржей, где последняя выступает в роли источника сырья. При получении оценки были использованы разные методы моделирования: смешано-целочисленное линейное программирование составление модели, которая находит оптимальные вектора раскроя, закупок сырья, производства товаров и способа транспортировки по графу, метод Монте Карло - генерирование начальных условий и параметров, имитационное моделирование - для каждого состояния решалась искомая поставленная задача. Основная вычислительная проблема модели заключается в значительном потребном объеме оперативной памяти компьютера для проведения исследования. Практическая значимость исследования заключается в доказательстве целесообразности закупа предприятием сырья с товарно-сырьевой биржи России. Теоретическая значимость исследования заключается в разработке новой модели по оценке целесообразности проведения закупок сырья с применением аппарата биржи. Научная новизна заключается в построенной математической модели формирования цепочек поставок, составления плана раскроя и объемов производства с учетом спроса на рынке и доступных объемов сырья. Апробация модели проведена на данных одного из предприятий лесопромышленной отрасли Приморского края. Оптимизация ведется по объему производимой продукции и закупаемого сырья из каждого региона, по количеству раскроев каждой из плит ОСП и по запасам сырья на складе производства. На основе апробации модели на данных биржи и предприятия лесопромышленной отрасли была дана оценка целесообразности сотрудничества компании и товарно-сырьевой биржи, которая отражает, что в 95 \% случаев имеется высокий потенциал сотрудничества.

Ключевые слова: задача раскроя, цепочки поставок, сырье, лесопромышленная отрасль, оптимальный план, производства, математическое моделирование.
\end{abstract}

Рогулин Родион Сергеевич e-mail: rafassiaofusa@mail.ru

() () Контент доступен под лицензией Creative Commons Attribution 4.0 License.

The content is available under Creative Commons Attribution 4.0 License. 


\section{Р. С. Рогулин}

\section{ВВЕДЕНИЕ}

Зарождение задачи формирования цепочек поставок совпало с появлением первого производства, когда возникла проблема бесперебойных поставок сырья. Важность эффективного поиска решения этой задачи впервые резко выросла в XVIII-XIX столетии, когда в Европе и мире проходила промышленная революция. Произошло резкое увеличение радиуса отправки ресурсов с предприятий и отправки ресурсов с пунктов их добычи, что в свою очередь усилило роль конкуренции на рынке. Теперь стандартный комплексный вопрос производства о том, где купить сырья, в каком объеме производить продукцию и кому из покупателей ее отправлять, стал нетривиальным за счет увеличения объемов информации, подлежащий обработке. Возникла серьезная потребность в развитии методов и подходов для решения такой задачи.

Для лесопромышленной отрасли народного хозяйства эта задача еще и усложнена тем, что необходимо знать, как проводить раскрой полученного сырья (круглого леса, далее круглого) так, чтобы цель предприятия была достигнута (минимизация отходов, максимизация прибыли и т. д.). Таким образом, количество вариантов по решению такой комплексной задачи растет крайне быстро, процесс поиска из множества допустимых решений затрудняется, что сказывается на затрачиваемом объеме времени, отводимом на поиск оптимального решения.

Сегодня не стоит забывать о том, что с течением времени количество лесов в мире не увеличивается (Forest Area (\% of land area) (1990-2016). The World Bank (WB) Data), население мира растет (Population, total (19602019). The World Bank (WB) Data), а следовательно, и спрос на товары, включая, лесопромышленной отрасли имеет все необходимые условия не падать (Forestry Production and Trade (1993-2019). Food and Agriculture Organization of the United Nations (FAO)). Отсутствие единых методов и подходов в решении данной задачи также не упрощает работу менеджерам предприятий в решении своих повседневных вопросов управления предприятием. Все это делает вопрос об оптимальном принятии решений на предприятии лесопромышленной отрасли нетривиальным и актуальным по настоящий момент.

\section{1. ОБЗОР ЛИТЕРАТУРЫ}

Рассмотрим мировой опыт в решении описываемой проблемы. Авторы работ [1-9] утверждают, что в рамках исследуемых отраслей промышленности ключевым фактором экономической эффективности работы предприятий являются вопросы оптимального управления и планирования. Так Kondili et. al. в статье [1] рассматривал вопросы оптимизации планирования в химической промышленности. Работа Kondili et. al. отличалась тем, что он предложил использовать многоцелевую оптимизационную модель планирования работы производства, где одним из ее отличительных шагов было внедрение так называемой концепции «Сети Государственных Задач» (State Task Network, далее STN), которая характеризуется отсутствием заранее известного назначения каждого станка на определённый вид работы. В недавней работе [2] другой коллектив ученых отметил работу [1], как одну из самых первых в области оптимизации процессов производства, планирования и доставки с учетом специфики работы в химической промышленности. В 2007 году научный коллектив в статье [3] отмечают, почему работа [1] не нашла широкого применения в промышленности. А именно ввиду того, что полученная модель была крайне большой по размерности и, как следствие, требовала значительных объемов оперативной памяти, что в то время было недопустимо.

Творческой переработкой использования идеи STN стала работа [4], где авторы заменили несколько типов Big-M ограничений на линейные, но более жесткие с точки зрения математического моделирования, что привело к уменьшению росту числа ограничений при добавлении одного дополнительного дня в периоде планирования. 
Влияние подзадачи о раскрое при оценке иелесообразности проведения закупок лесного сырья ...

В рамках процесса планирования важную роль играет длина горизонта планирования. В работе [5] отмечается, что при достаточно большой длине горизонта планирования возникают проблемы размерности, которые можно решать путем комбинирования подходов моделирования течения времени в виде дискретных и непрерывных величин.

В недавней работе [6] Castro и Mostafaei решали проблему планирования транспортировки жидкости. Предложенная модель была сформулирована в рамках смешано-целочисленного линейного программирования (Mixed-Integer Linear Programming, далее MILP), которая описывается, как сеть возможных событий, где один входной узел ведет к множеству вероятностных выходов. Здесь было использовано непрерывное представление времени и адаптированы новые ограничения для предотвращения запрещенных последовательностей продуктов.

На 28-м Европейском симпозиуме по автоматизированному проектированию процессов (28th European Symposium on Computer Aided Process Engineering) была доложена работа [7] о планировании этапов стерилизации и упаковки в крупномасштабном производстве рыбных консервов в Испании. Обсуждался алгоритм декомпозиции с применением модели MILP, который использовался для сокращения требуемых вычислительных мощностей, поскольку продукты загружаются в контейнеры итеративным способом, пока не будет сформировано окончательное расписание. Модель общего приоритета эффективно описывает периодический (стерилизация) и непрерывный (упаковка) процессы на предприятии. Общее время поиска решения при апробации алгоритмы не превышал 20 минут.

На 29-м Европейском симпозиуме по автоматизированному проектированию процессов обсуждалась работа [8], которая была посвящена методу скользящего горизонта. Он был встроен для оперативной корректировки графика поставок в случае сбоев, таких как отмена или изменение заказов, или внезапное поступление новых заказов, или любые отклонения от запланированного производства. 158 готовых продуктов (сгруппированных в 44 семейства продуктов) были распределены на шести параллельных упаковочных линиях, а интересующий временной горизонт составлял пять дней. Общее снижение затрат на 20 \% было достигнуто по сравнению с изначальными результатами, полученными компанией по исходному алгоритму. На том же симпозиуме Elekidis et al. доложили результаты исследования по решению проблемы о краткосрочном планировании доставки потребительских товаров ритейлинговой компанией [9]. Модель использует концепцию «общего приоритета», в которой основное внимание уделялось стадии упаковки. Ограничения учитывали объем удовлетворенного спроса с предыдущих этапов, что позволяло проводить комплексную оптимизацию на всем горизонте планирования. Произведенные и упакованные заказы входят в контейнер итеративно с использованием алгоритма декомпозиции. Были рассмотрены различные примеры реальных исследований, которые включают до шести упаковочных линий и 130 готовых продуктов. Генерируются субоптимальные расписания и вычисляются объемы экономии времени на переключении планов.

В статье [10] авторы рассматривали проблему закупку сырья с товарно-сырьевой биржи. Особенность ее заключается в том, что в процессе транспортировки может произойти отказ от купленной партии лесного сырья, если последнее идет дольше, чем это обсуждено в договоре купли-продажи, что нарушает производственный цикл предприятия. Авторы сформулировали математическую модель нелинейного целочисленного стохастического программирования, которая учитывает этот вид риска. В качестве алгоритма поиска решения использовался авторский алгоритм, разработанный на базе генетического алгоритма и метода ветвей и границ.

Авторы работы [11] разработаны две модели MILP, учитывающие большое множество эксплуатационных, проектных и отраслевых ограничений пищевой промышлен- 


\section{Р. С. Рогулин}

ности. В непрерывных временных рамках используется общий приоритет или подход общего приоритета для конкретных единиц в зависимости от данной цели, в то время как сетка с дискретным временем используется для наложения технико-экономических ограничений, связанных с этапом пакетной обработки. Алгоритм декомпозиции применяется для обеспечения решения задачи планирования в течение желаемого времени вычислений. В этом методе продукты, которые нужно запланировать, оптимизируются итеративно в соответствии с определенной пользователем политикой вставки.

В работе [12] авторы исходя из предпосылки удовлетворения потребностей клиентов, чтобы повысить прибыль предприятий, в качестве основы целевой функции используют следующие параметры: стоимость, время и качество. Делается предположение, что больше всего беспокоят клиентов эти три фактора. Для поиска решения была построена многокритериальная математическая модель для планирования производства и создания предприятия в облачной производственной среде с наименьшими затратами, работающее за кратчайшие сроки и с максимальным уровнем качества. Ввиду недостатков традиционной многокритериальной задачи оптимизации алгоритм NSGA-II используется для решения указанной выше многокритериальной модели оптимизации.

Особенность статьи [13] заключается в том, что, разработанная математическая модель, сформулирована с применением режима отсутствия счета-фактуры и режима непроданного объема товаров и стратегии предложения продукта для модели «реального спроса» с использованием соответствующих принципов теории вероятностей, уравнений в частных производных и теории оптимизации. Данные особенности модели могут помочь производителям или розничным продавцам принимать более точные решения о продажах продукции для каждого в отдельности покупателя и групп клиентов в соответствии с различными моделями спроса.

В работах ушедшего 2020-го года [14-21] часто обсуждается, что для производств из перерабатывающих отраслей высокую актуальность имеют задачи раскроя.

Так в статье [22] описывается алгоритм, разработанный для решения частной проблемы с раскроем материалов, возникающей на бумажной фабрике в Пешаваре. Основная цель разработанного решения - минимизировать потери при обрезке, возникающие при производстве и выделки мастер-катушек различной ширины и пригодных для использования объемы остатков при соблюдении ряда эксплуатационных и технологических ограничений. Для этого была разработана карта раскроя. Полученная карта раскроя удовлетворяет наложенным операционным и технологическим ограничениям и используется в качестве столбцов-переменных модели линейного программирования. Алгоритм поиска решения поделен на два этапа: с применением симплекс-метода и специального, суть которого заключается в поиске целочисленного решения, если изначально полученное содержит нецелые переменные решения.

В исследовании [23] проводится двухэтапная математическая модель планирования стратегии раскроя листов на основе улучшенного генетического алгоритма. Метод раскроя представлен четырехмерным вектором, который сводится к одномерному. Затем с использованием генетического алгоритма происходит расчет субоптимальных значений модели.

В статье [24] генетический алгоритм используется в качестве основного алгоритма для реализации системы автоматического планирования по раскрою двумерных пластин в сочетании с технологическими требованиями. Согласно практическим исследованиям на рынке строительных материалов, определенный тип листового материала и конечный вид продукта моделируются с точки зрения различных требований, в которой также рассчитываются и прогнозируются коэффициент прибыли поставщиков. Результаты показывают, что по сравнению с другими методами оптимальная стратегия раскроя листа, основанная на улучшенном генетическом алгоритме, снижает вычислительную сложность при условии высокой загрузки, что бо- 
Влияние подзадачи о раскрое при оценке целесообразности проведения закупок лесного сырья ...

лее подходит для систем с различными типами продуктов и их количеством ограничений.

Исходя из проведенного обзора можно утверждать, что задачи производства для разных отраслей промышленности имеют свои особенности, которые необходимо учитывать при принятии решений. При всем многообразии имеющихся работ, посвященных по отдельности четырем основным проблемам производства (формирование цепочек поставок сырья и готовой продукции, оптимальные вектор производства товаров, вектор закупок сырья и план раскроя), слабо представлены исследования, которые бы решали все четыре задачи в комплексе.

\section{2. ЦЕЛИ И ЗАДАЧИ ИССЛЕДОВАНИЯ}

Целью исследования является разработка экономико-математической модели по оценке целесообразности взаимодействия предприятия в долгосрочной перспективе с товарно-сырьевой биржей на аукционных торгах.

Выдвигаются следующие задачи для достижения цели исследования:

- Разработать модель, позволяющую максимизировать доналоговую прибыль предприятия на основе: изменения цен и спроса на конечную продукцию в определенных границах, изменения норм объемов сырья потребных для производства каждой единицы продукции, оптимизации планирования закупок сырья с биржи и объёмов производства с использованием уже реализованных заявок на товарно-сырьевой бирже за прошедший период и отличающуюся возможностью оптимизации объемов раскроя круглого;

- Апробировать модель в условиях реального предприятия и исследовать вычислительные аспекты применения модели;

- Провести экономический анализ полученных результатов в ходе работы модели и дать оценку целесообразности работы предприятия с биржей.

Сформулируем гипотезу исследования исходя из цели и задач исследования: взаимодействие предприятия с товарно-сырьевой биржей России носит выгодный характер.

\section{3. МАТЕМАТИЧЕСКАЯ МОДЕЛЬ}

Главной проблемой производственных комплексов является невозможность их работы в условиях отсутствия сырья. Отсюда вытекает большая задача о формировании устойчивых цепочек поставок сырья. При наращивании запасов сырья на складе можно пользоваться услугами: товарно-сырьевых бирж, предпринимателей из соседних к комплексу делян или эксплуатировать деляны, полученные в аренду. В рамках данной работы сосредоточимся на взаимодействии с биржами (с Санкт-Петербургской Международной Товарно-сырьевой Биржей (АО «СПбМТСБ»)), т. к. существует достаточно большое количество научных работ по взаимодействию предприятий и частников, предприятий и эксплуатируемых арендуемых ими же делян. Однако, при взаимодействии с биржами возникает вопрос о том, из какого региона в какой день и в каком объеме производить закупку сырья. На бирже каждый день публикуются все вышеупомянутые данные о совершенных сделках. Кроме того, биржа оказывает услуги по доставке сырья до потребителя, что также включается в цену товару. На основе публикуемых данных построим модель по имитации взаимодействия предприятия с товарно-сырьевой биржей для расчета оценки целесообразности закупа сырья с бирж.

После доставки сырья на склад технология производства требует измельчения круглого до состояния трухи и изготовки из нее OSB-1 (ОСП-1) плит (Ориентированно-стружечная плита) (далее плит). Длина, высота и ширина плит, как правило, фиксированы.

Следующей задачей производства является проблема поиска оптимального плана раскроя плит на заготовки определенной длины и об объеме производства из заготовок конечных видов товаров. Предполагается, что при раскрое плиты по длине можно дальше проводить раскрой по ширине, однако, мы сосредоточимся на раскрое только по длине (Сделаем предположение, что из полученных раскроев по длине при дальнейшем заранее 


\section{Р. С. Рогулин}

технологически оговоренным раскрое по ширине можно получить все необходимые детали этой длины для изготовки конечного вида товара. Следовательно, остается лишь вопрос о способе проведения раскроя плиты по длине так, чтобы получить заготовок необходимых длин достаточное количество для получения потребного для производства количества деталей этой же длины.). Однако, объем раскроев необходимо спланировать так, чтобы хватило всех длин заготовок на производство каждого вида товаров.

Также стоит помнить о спросе на рынке товаров, который нужно по возможности максимально удовлетворить. Чтобы доставить до потребителя товары необходимо составить план доставки от пункта производства до потребителя по заранее известному дорожному графу (дорожная карта).

Для решения поставленной задачи необходимо сформулировать комплексную модель оптимизации всех вышеописанных процессов:

$M$ - рассматриваемый горизонт планирования (дни) [21];

$k-$ тип производимой продукции, $k=1, \ldots, K[21]$;

$l e n_{e}-$ длина заготовки типа $e$, м;

width, height - ширина и толщина заготовок соответственно, м;

$w(m)$ - номер недели $w$ в зависимости от номера дня $m$, где

$$
w(m)=\left\{\begin{array}{l}
\left\lceil\frac{m}{7}\right\rceil, \quad \text { если } m=M \\
\frac{m-1}{7}+1, \text { если }\left\{\begin{array}{l}
\left\lceil\frac{m-1}{7}\right\rceil-\left\lfloor\frac{m-1}{7}\right\rfloor=0 \\
\left\lceil\frac{M}{7}\right\rceil-\left\lceil\frac{m}{7}\right\rceil>0
\end{array}\right. \\
\nexists, \quad \text { иначе }
\end{array}\right.
$$$$
W(M)=\left\lceil\frac{M}{7}\right\rceil \text { - количество недель } W \text { в }
$$
зависимости от горизонта планирования $M$;

$c_{i m r l}$ - цена покупки $1 \mathrm{~m}^{3}$ из $i$-й заявки сырья $l$-го типа в $r$-м регионе в $m$-й день (руб). Стоимость доставки включена [21]; $c_{j}$ - затраты на транспортировку в пункт $j$ (руб);

$b_{e t}$ - количество раз, когда встречается заготовка типа $e$ в раскрое типа $t\left(\left\{b_{e t}\right\}-\right.$ карта раскроя);

$V_{i m r l}$ - объем сырья типа $l$ в заявке $i$ из региона $r$ в день $m\left(\mathrm{M}^{3}\right)$ [21];

$\bar{u}$ - максимальная вместимость склада $\left(\mathrm{M}^{3}\right)[21]$;

$\bar{O}$ - максимальное количество раскроев в день (ед.) [21];

$v_{i m r l}^{\prime}$ - объемы сырья типа $l$, купленные в предыдущем периоде, про которые известно, что они поступят на склад в день $m\left(\mathrm{M}^{3}\right)$ [21];

$p_{k m l}$ - цена продажи товара типа $k$ в день $m$ изготовленного из сырья типа $l$ за вычетом стоимости основного используемого сырья (лесоматериалы, затрат на общие нужды (свет, вода, клей и пр.)) (руб) [21];

$I^{\prime}-$ количество заявок, которые были куплены в предыдущий период (до $m=0$ ) и дата их прихода на склад заранее известна [21];

$R$ - количество регионов;

$T_{r}$ - норма временных затрат (в днях) на доставку любого объема сырья из региона $r$ по ж/д [21];

$Q_{j k w l}-$ спрос розничной компании $j$ на товар $k$ из ресурса типа $l$ на неделе $w$;

$\dot{Q}_{j k w l}$ - математическое ожидание объема спроса на продукцию типа $k$ из ресурса типа $l$ в неделю $w$ розничной компанией $j$;

$J^{*}$ - множество розничных торговцев (конечные пункты назначения произведенных типов товаров);

$B u d_{0}$ - начальный бюджет предприятия; month $(m)$ - номер месяца month в зависимости от номера для $m$;

$A_{\text {ekl }}^{\text {month }(m)}$ - количество затрат заготовок типа $e$ на производство единицы товара $k$ из peсурса типа $l$ по технологии в месяц month $(m)$ (ед.) [21];

iter - количество независимых итераций (ед.) [21];

$F C$ - фиксированные постоянные издержки (руб) [21];

$V$ - объем плиты $\left(\right.$ м $\left.^{3}\right)$;

$\tilde{L}$ - длина плиты (м);

$T_{r}$ - время доставки сырья из региона $r$ (дни); 
Влияние подзадачи о раскрое при оценке иелесообразности проведения закупок лесного сырья ...

$x_{k m l}-$ объем производства товаров типа $k$ в день $m$ изготовленного из сырья типа $l$ (шт) $[21]$;

$z_{j k w l}$ - объем транспортировки до пункта $j$ товара типа $k$ из сырья типа $l$ (ед.) на неделе $w(m)$;

$q_{t m l}$ - количество раскроев типа $t$ в день $m$ из сырья типа $l$;

$v_{i m r l}$ - покупаемый объем сырья типа $l$ из заявки $i$ из региона $r$ в день $m\left(\mathrm{M}^{3}\right)$ [21];

$u_{m l}$ - запас на складе сырья типа $l$ в день $m\left(\mathrm{M}^{3}\right)[21]$;

$\tilde{u}_{e m l}$ - объем запаса заготовок типа $e$ ко дню $m$ из сырья $l$.

В качестве целевой функции будем рассматривать значение доналоговой прибыли предприятия на горизонте планирования $M(2)$ :

$$
\max _{x, v, z} \sum_{m}\left(\sum_{k} p_{k m l} x_{k m l}-\sum_{i, l, r} c_{i m r l} v_{i m k l}-\sum_{j, k, l} c_{j} z_{j k w(m) l}\right)
$$

При ограничениях (3-16):

$$
\begin{aligned}
& \tilde{u}_{e m l}=\tilde{u}_{e(m-1) l}+\sum_{t} b_{e t} q_{t m l}-\sum_{k} A_{e k l}^{m o n t h(m)} x_{k m l} \text {, } \\
& e=1: E, m=1: M, l=1: L \\
& \sum_{t, l} q_{t m l} \leq \bar{O}, \quad m=1: M \\
& \sum_{m=7 *(w-1)+1}^{7 * w} x_{k m l} \leq \sum_{j} z_{j k w l}, \\
& m=1: M, k=1: K, l=1: L \\
& z_{j k w l} \leq Q_{j k w l}, \quad j=1: J^{*}, k=1: K, \\
& w=1: W, l=1: L \\
& \sum_{t}\left(u_{m l}+\sum_{e} \tilde{u}_{e m l} * \text { len } n_{e} * \text { height } * \text { width }\right) \leq \bar{u}, \\
& m=1: M \\
& x_{k m l}, q_{t m l}, z_{j k w l} \in Z^{+} \\
& \tilde{u}_{e m l}, u_{m l}, v_{i m r l} \geq 0 \\
& v_{\text {imrl }} \leq V_{\text {imrl }}, i=1: I, m=1: M, r=1: R, l=1: L \\
& B u d_{0}+\sum_{m=1}^{m^{*}}\left(\sum_{k, l} p_{k m l} x_{k m l}-\sum_{i, r, l} c_{i m r l} v_{i m r l}-\right. \\
& \left.-\sum_{j, k, l} c_{i} z_{j k w(m) l}-F C\right) \geq 0, m^{*}=1: M \\
& u_{m l}=u_{(m-1) l}+\sum_{i, r} v_{i\left(m-T_{r}\right) r l}-V \sum_{t} q_{t m l} \\
& V, B_{u d}, \tilde{u}_{e 0 l}, A_{e k l}^{\text {month }(0)}=\mathrm{const}
\end{aligned}
$$

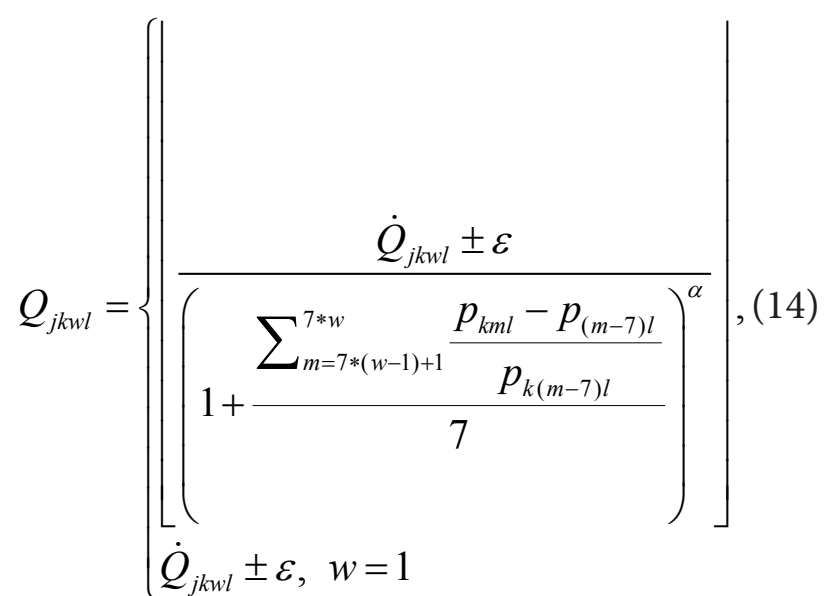

$$
\begin{gathered}
w=2: W, j=1: J^{*}, k=1: K, l=1: L \\
p_{k(m+1) l}=p_{k m l} *\left(1+\varepsilon^{1}\right), \\
m=1: M-1, \varepsilon^{1}=\left[-\varepsilon_{1}^{1} ; \varepsilon_{2}^{1}\right] \\
A_{e k l}^{\text {month }(m)}=\max \left(0 ; \min \left(A_{e k l}^{\text {month }(m)}, A_{e k l}^{\text {month }(m)}+\varepsilon^{2}\right)\right), \\
\varepsilon^{2}=\left[-\varepsilon_{1}^{2} ; \varepsilon_{2}^{2}\right]
\end{gathered}
$$

где $\varepsilon, \varepsilon^{1}, \varepsilon^{2}$ - равномерно распределенные случайные величины непрерывного типа, $\varepsilon_{1}^{1}$, $\varepsilon_{2}^{1}, \varepsilon_{1}^{2}, \varepsilon_{2}^{2}, \alpha=$ const.

Рассмотрим подробнее ограничения (3)(16). (3) отражает объем на складе запасы заготовок на всем горизонте планирования. Ограничение (4) утверждает максимальное количество листов для раскроя в день. (5) ограничивает сверху объем производимого количества товаров каждую неделю объем транспортировки, но при целевой функции (2) можно считать, что (5) превращается в равенство. Ограничение (6) утверждает, что объем транспортировки до конечных пунктов не может превышать в них объем спроса. (7) ограничивает сверху состояние заполненности склада. (8)-(9) задают характер переменных. Объем закупаемого сырья каждый день ограничивается размером заявки на бирже (10). (11) отражает объем прибыли каждый день, который должен быть неотрицательным. (12) утверждает запас сырья на складе. (13) отражает стартовые и постоянные значения параметров. (14) утверждает зависимость спроса от изменения цены во времени. (15) - есть рекуррентная зависимость цены от номера дня. (16) отражает нормы потребления заготовок на производство каждой единицы товара. 


\section{Р. С. Рогулин}

Рассмотрим некоторые особенности данной модели. Данная задача (2)-(16) при разыгранных значениях (14)-(16) относится к класcy MILP. Для достижения цели исследования будем решать задачу (2)-(14) при разыгранных значениях (14)-(16), как задачу MILP. Быстрое изменение количества переменных при добавлении хотя бы одного дня. Большое количество ограничений, в которых участвует большое количество переменных, увеличивает время поиска первого допустимого решения. Некоторые переменные носят целочисленный характер. Большое количество независимых итераций при поиске решений.

В качестве среды программирования выберем Matlab. В качестве алгоритма выберем смешанный: метод Гоморри и Ветвей и границ (MathWorks. Documentation. Intlinprog), т. к. он максимально оптимизирован в среде программирования Matlab. Краткое описание этого алгоритма изложена в работе [21, c. 497].

\section{4. ТЕСТИРОВАНИЕ МОДЕЛИ И КАЛИБРОВКА}

Проведем тестирование модели на данных Санкт-Петербургской Международной Товарно-сырьевой Биржи. В конце каждого дня торгов публикуются данные о совершенных сделках. На базе этой статистике проведем оценку целесообразности взаимодействия одного из крупных и одновременно молодых предприятий Приморского края. Причина проведения этой оценки вызвана тем, что сырья на рынке Приморского края одно из самых дорогих в стране, т. к. в непосредственной близости находится крупный оптовый покупатель в лице КНР [20].

Для апробации модели воспользуемся собранной выборкой с биржи за 11 месяцев (с 01 февраль 2020 года по 30 ноября 2020 года), другими словами, за 303 дня $(M)$, суммарно по всем интересующим предприятие видам сырья было опубликовано 2145 заявок. Исходя из официальной статистики биржи за это за обозначенный выше временной интервал ее работы участвовали предприятия по лесо- заготовке из 5-ти регионов в качестве продавцов сырья: Иркутская область $(r=1)$, Республика Удмуртия (далее, Удмуртия $(r=2))$, Московская область $(r=3)$, Пермский край $(r=4)$, Республика Бурятия (далее, Бурятия $(r=5))$. Т. к. биржа скрывает реальные названия и местоположения продавцов, то более детальная информация по продавцам отсутствует. За обозначенный период был получен массив следующих данных с предприятий и биржи: цены $\left(c_{i m r l}\right)$, даты $(m)$ появления сырья, объемы $\left(v_{i m r l}\right)$ в эти дни, цены заявок $\left(p_{k m l}\right)$, количество заявок по каждому типу сырья. Кроме того, известен спрос $\dot{Q}_{j k w l}$ в каждую неделю каждого вида товара исходя из статистики продаж предприятия.

Кроме того, предприятие имеет некоторые начальные и технологические данные, изложенные в табл. 1-3.

Карта раскроя составлялась с применением известного алгоритма [24].

Нормозатраты сырья на производство каждой единицы товара представлены в табл. 3.

Принято считать, что цена каждый день не может меняться более, чем на три процента. В табл. 4 отражены значения случайных величин.

Для поиска решения воспользуемся языком программирования Matlab, а именно функцией intlinprog. Задача характеризуется достаточной размерностью, что не позволяет утверждать, что оптимальное решение будет найдено за короткое или допустимое время, поэтому было решено ограничить количество проходимых вершин множества допустимых решений в размере $10^{7}$ ед. В случае, если допустимое решение было найдено, но алгоритм вышел из цикла в связи с описанным выше ограничением, будем считать, что решение не носит характер оптимального, а носит условно характер эфбективного. В качестве технологии параллельного программирования воспользуемся MPI, также встроенной в Matlab. Количество итераций ограничим 11000 , что означает, что на каждый день из периода планирования приходится примерно 34 итерации.

Отдельно отметим, что в связи с корпоративной этикой в работе не указывается реаль- 
Влияние подзадачи о раскрое при оценке целесообразности проведения закупок лесного сырья ...

Таблица 1. Входные начальные и постоянные данные

[Table 1. Input initial and constant data]

\begin{tabular}{|l|l|}
\hline \multicolumn{1}{|c|}{ Параметр, ед. измерения } & \multicolumn{1}{|c|}{ Значение параметра } \\
\hline $\bar{u}, \mathrm{M}^{3}$ & 7500 \\
\hline $\bar{O}$, ед. & 3000 \\
\hline $\begin{array}{l}u_{0 l}, l=1: 2, \mathrm{M}^{3}(\text { На начальный момент }(m=0) \\
\text { работы алгоритма (входные данные) }\end{array}$ & {$[2250,2250]$} \\
\hline$p_{k m l}, m=1, \forall l$, т. р. & {$[22.1,32.8,40.8,43.7,45.5,48.5,57.5,60.5,66.9]$} \\
\hline$T_{r}$, дни & {$[4,5,7,5,5]$} \\
\hline$B u d_{0}$, руб. & 10000000 \\
\hline$F C$, руб. & 1000000 \\
\hline$\alpha$ & 2 \\
\hline$V, \mathrm{M}^{3}$ & 0.4 \\
\hline$\tilde{u}_{e 0 l}, \forall l$, ед. & 400 \\
\hline$h e i g h t \times w i d t h, \mathrm{M}^{2}$ & $2 \times 2$ \\
\hline$l e n_{e}, e=1: E, \mathrm{M}$ & {$[0.5,0.55,0.115,0.125]$} \\
\hline
\end{tabular}

Источники: ООО «ДНС-Лес», автор.

Таблииа 2. Карта раскроя листа длиною $\tilde{L}$

[Table 2. Cutting chart for sheet length of $\tilde{L}$ ]

\begin{tabular}{|c|c|c|c|c|c|c|c|c|c|}
\hline$e$ & len $_{e} /$ номер раскроя & 1 & 2 & 3 & 4 & 5 & 6 & 7 & 8 \\
\hline 1 & 0.5 & 4 & 2 & 1 & 1 & 1 & 0 & 0 & 0 \\
\hline 2 & 0.55 & 0 & 1 & 2 & 0 & 0 & 3 & 1 & 1 \\
\hline 3 & 0.115 & 0 & 0 & 0 & 1 & 0 & 0 & 1 & 0 \\
\hline 4 & 0.125 & 0 & 0 & 0 & 0 & 1 & 0 & 0 & 1 \\
\hline & Отходы, м3 & 0 & 0.45 & 0.4 & 0.35 & 0.25 & 0.35 & 0.3 & 0.2 \\
\hline
\end{tabular}

Источники: Составлено автором.

Таблица 3. Нормозатраты заготовок на производство каждой единицы продукции $k$ из сырья muna $l$

[Table 3. The standard costs of blanks for the production of each unit of product $k$ from raw materials of type 1$]$

\begin{tabular}{|l|c|c|c|c|c|c|c|c|c|c|}
\hline$l$ & $e / k$ & 1 & 2 & 3 & 4 & 5 & 6 & 7 & 8 & 9 \\
\hline \multirow{4}{*}{1} & 1 & 0 & 1 & 1 & 1 & 2 & 2 & 1 & 2 & 2 \\
\cline { 2 - 11 } & 2 & 0 & 1 & 0 & 1 & 2 & 2 & 1 & 2 & 2 \\
\cline { 2 - 11 } & 3 & 1 & 2 & 1 & 2 & 2 & 1 & 0 & 1 & 2 \\
\cline { 2 - 11 } & 4 & 1 & 2 & 1 & 2 & 1 & 2 & 1 & 1 & 1 \\
\hline \multirow{3}{*}{2} & 1 & 0 & 2 & 1 & 0 & 2 & 1 & 0 & 1 & 0 \\
\cline { 2 - 11 } & 2 & 0 & 2 & 0 & 1 & 2 & 2 & 2 & 2 & 2 \\
\cline { 2 - 11 } & 3 & 1 & 0 & 0 & 2 & 0 & 1 & 1 & 1 & 2 \\
\cline { 2 - 11 } & 4 & 1 & 2 & 2 & 2 & 1 & 2 & 1 & 2 & 1 \\
\hline
\end{tabular}

Источники: ООО «ДНС-Лес». 
Таблииа 4. Значения диапазонов случайных величин

[Table 4. Stochastic values intervals]

\begin{tabular}{|c|c|c|c|c|c|c|}
\hline Параметры & $\varepsilon_{1}^{1}$ & $\varepsilon_{1}^{2}$ & $\varepsilon_{2}^{1}$ & $\varepsilon_{2}^{2}$ & $\varepsilon$ & $\alpha$ \\
\hline Значения & 0.03 & 0.03 & 1 & 10 & 2 & 3 \\
\hline
\end{tabular}

Источники: ООО «ДНС-Лес», автор.

ный зафиксированный спрос $\dot{Q}_{n k m}$. Однако, данные касательно спроса ave $\left(\sum_{n}^{n} Q_{n k m}\right)$ представлены на рис. 1 (Под оператором ave(...) будем понимать округленные средние значения за все итерации).

\section{5. РЕЗУЛЬТАТЫ ИССЛЕДОВАНИЯ}

На поиск решений для всех итераций было задействовано почти четыре часа и более 14 Гб оперативной памяти компьютера. Отметим также, что использовались значительные вычислительные мощности в виде современного процессора с частотой до 4.2 ГГц с 12-ю потоками. Такой долгий процесс поиска решений при работе мощного процессора позволяет сделать вывод о высоких требованиях к компьютерным мощностям при решении искомой задачи. 87 \% решений носит характер оптимальных.

Рассмотрим рис. 1. Черным и серым цветами отражены динамики изменения средних цен каждого типа сырья по всем итерациям. Из рис. 1 следует, что в среднем спрос падает к лету, а после него наблюдается неуверенный рост. Заметим, что средний спрос на каждый технологически более сложный товар меньше предыдущего, но «провал» спроса тем меньше, чем более сложным и требовательным к объему ресурсов товар является. Причины такого поведения описаны в работе [20].

Для того, чтобы опровергнуть или подтвердить гипотезу исследования стоит рассмотреть рис. 2, на котором отражена динамика поведения прибыли на всем горизонте планирования. Здесь видно, что условно процесс извлечения прибыли можно разделить на две стадии: наращивание объема прибыли и на сложный период. Рассмотрим последний подробнее. Сложность его заключается в том, что невозможно не производить товары, ина-
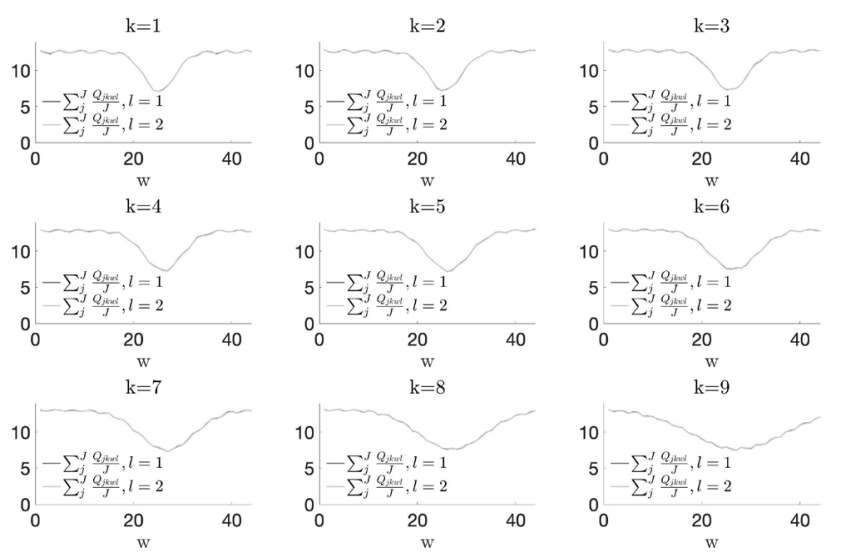

Рис. 1. Визуализачия объемов спроса

[Fig. 1. Demand quantity visualization]

че объем издержек будет слишком большой, но в то же время, процесс производства и реализации товаров не приводит к увеличению показателя прибыли. Первая же стадия характеризуется реальным периодом наращивания прибыли. Таким образом, самым сложным периодом планирования является июнь, июль и первая половина августа. Связано это c «провалом» спроса на все виды товаров, который отражен на рис. 1. Кроме того, можно сделать вывод о том, что при изменении цены всего на 3 процента вверх и вниз дисперсия конечного значения прибыли достаточно велика, что позволяет сделать промежуточный вывод об актуальности решения задачи о поиске оптимального вектора цен на каждый вид товара, выполненного из каждого вида сырья, на всем горизонте планирования хозяйственной деятельности предприятия в связке с функцией спроса. Однако ввиду того, что в более, чем $95 \%$ случаях значение прибыли выросло, можно считать, что перспектива сотрудничества предприятия лесопромышленной отрасли с товарно-сырьевой биржей носит положительный и эффективный характер.

В аналогичных работах $[20,21]$ не наблюдалась ситуация, когда значение прибыли в конце горизонта планирования не превосходило начального. Это связано с тем, что в работу добавлены факторы транспортировки продукции до покупателя и возможность раскроя плит. 
Влияние подзадачи о раскрое при оценке целесообразности проведения закупок лесного сырья ...

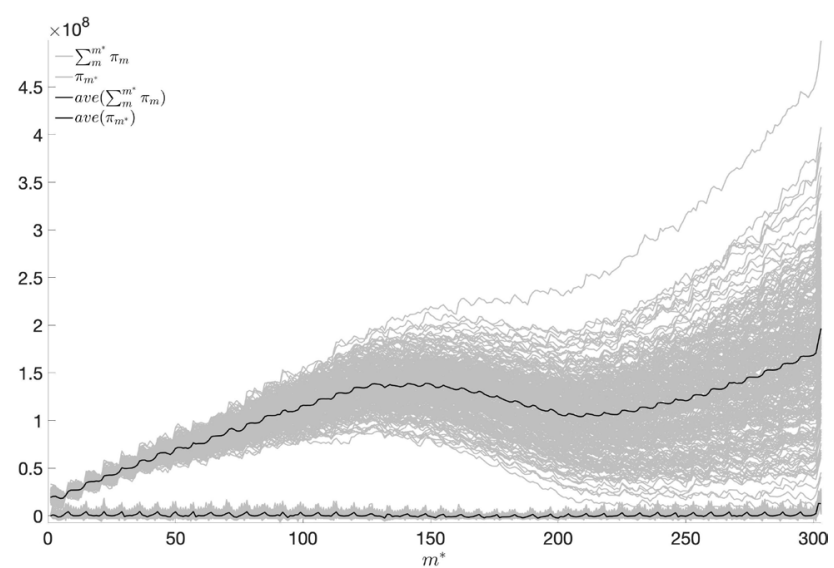

Рис. 2. Визуализация распределения значения прибыли во времени

[Fig. 2. Visualization of profit distribution in time]

Для более детального понимания всей картины, которая происходит на предприятии в ходе ее работы на заданном горизонте планирования необходимо рассмотреть ситуации, складывающиеся на складе и в цехах производства.

Начнем со склада, для этого рассмотрим поведение запасов заготовок на рис. 3. Основная масса заготовок изготавливается из первого типа сырья. Для второго типа сырья характерно, что примерно на 130 день на складе остается минимальный суммарный запас.
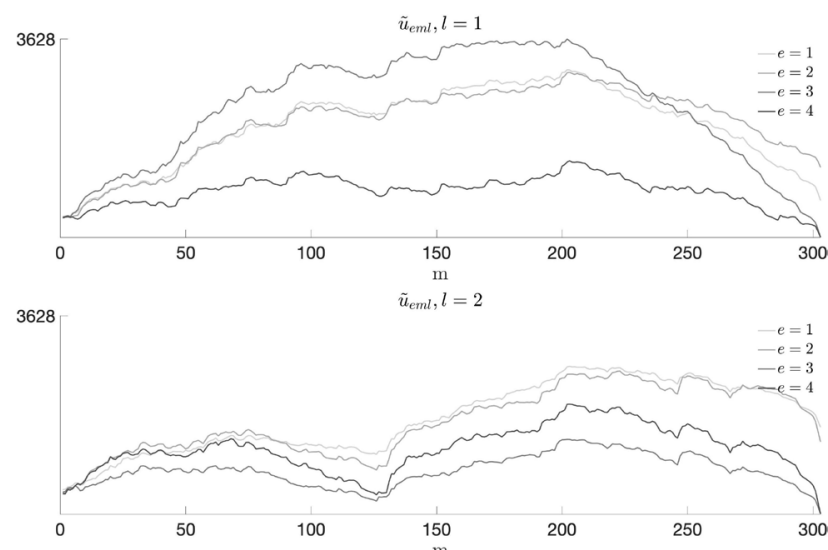

Рис. 3. Визуализация запасов заготовок на складе

[Fig. 3. Visualization of stock of blanks in the warehouse]

Рассмотрим объемы закупа сырья с биржи на рис. 4. Из него видно, что основная масса закупленного сырья обоих типов следует из Бурятии $(r=5)$. Однако, в работах [20, 21] для периода за 2019 год основной объем следовал из Иркутской области. Также можно заметить, что второго типа сырья поступает меньше.
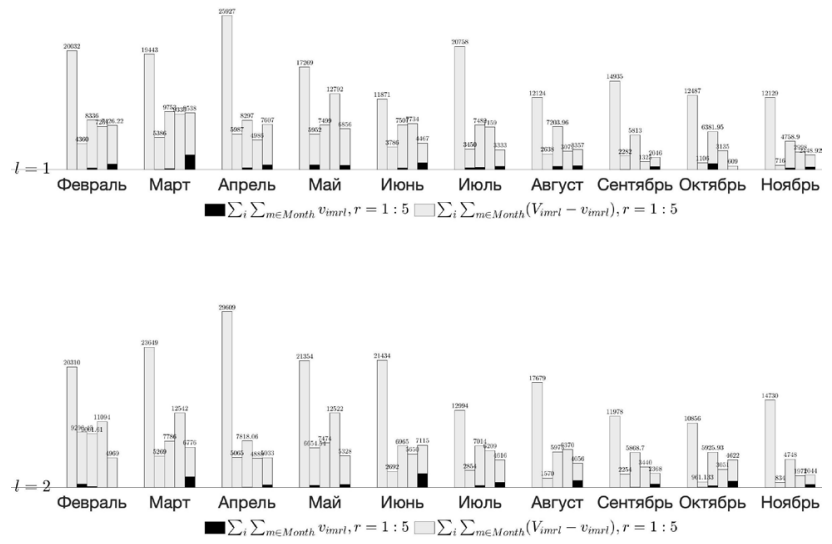

Рис. 4. Визуализация объемов закупа сырья из регионов

[Fig. 4. Visualization of quantity of bought row materials from Stock Exchange from regions]

Рассмотрим итоговую картину состояния склада на всем горизонте планирования на рис. 5. Отсюда видно, что 7500 - это довольно большой объем для склада. Основные периоды максимальной наполненности склада находятся в феврале, марте, апреле, июне, августе, сентябре. Пусть vol $_{e}$ - это объем заготовки типа $e$, тогда $v_{e} l_{e}=l e n_{e} *$ width $*$ height.

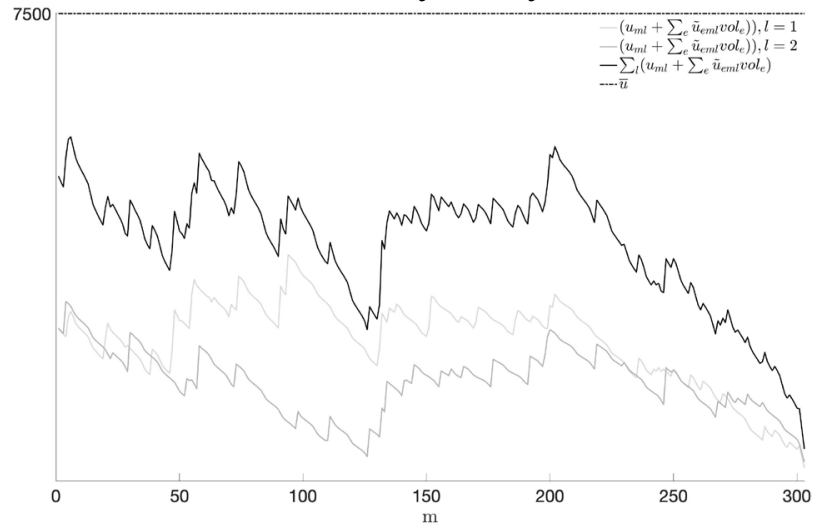

Рис. 5. Визуализаиия степени наполненности склада во времени

[Fig. 5. Visualization of the degree of warehouse fullness in time]

Рассмотрим объемы производства товаров. В июле наблюдается снижение объемов производства. Можно заметить, что все това- 


\section{Р. С. Рогулин}

ры производятся в примерно равной пропорции. В то время, как в работах $[20,21]$ в аналогичный период планирования - 2019 год, объемы производства товаров менялись по более сложному закону несмотря на аналогичную ситуацию со спросом.

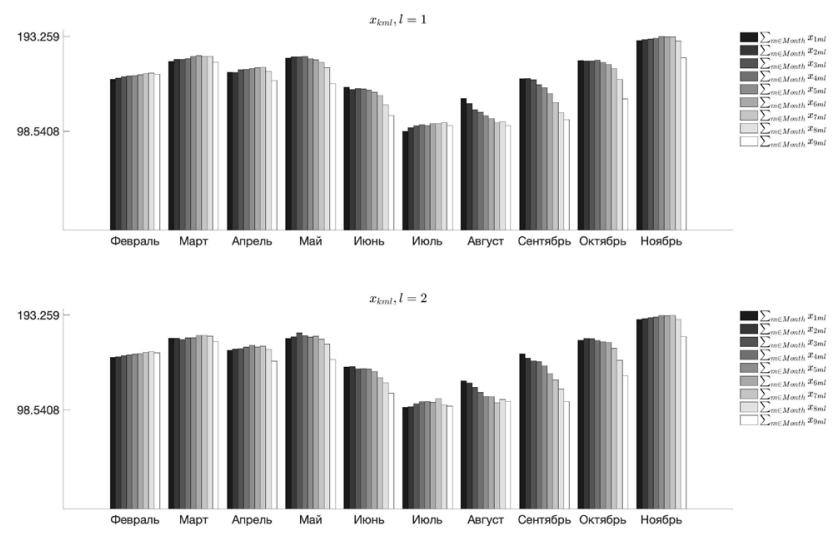

Рис. 6. Визуализация объемов производства по месяиам

[Fig. 6. Manufacture quantities by months visualization]

Рассмотрим отходы от плит, которые получаются в ходе раскроя на рис. 7. Их дальнейшая продажа или расщепление могли бы послужить дополнительным внушительной добавкой к доходу. Заметим, что для второго типа сырья характерно, что примерно на 130й день наблюдается минимальная дисперсия объемов накопленных отходов.
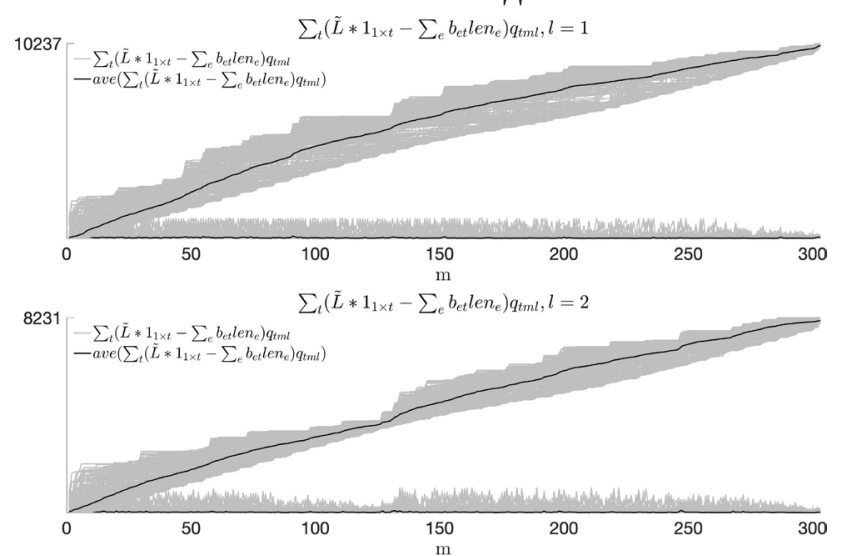

Рис. 7. Визуализация объемов отходов

[Fig. 7. Waste volume visualization]

Более детальный анализ положения на складе и в цехах также подтвердил гипотезу о положительной перспективе сотрудничества с биржей.
Рассмотрим возможности по модернизации модели. Можно сформулировать несколько самых важных вопросов производства лесопромышленной отрасли, которые не нашли отражения в рамках этой работы:

1. Вероятность отказа от сырья, если купленная заявка идет дольше, чем это описано в договоре о купли-продаже [20, 21].

2. Степень загруженности и структуру рассматриваемой транспортной сети $[20,21]$.

3. Возможность получения делян - источника сырья, в аренду для лесопользования $[20,21]$.

4. В случае отрицательной прибыли на каком-то интервале горизонта планирования необходимо предусмотреть возможности по получению инвестиций из-за пределов предприятия.

5. Возможность реализовывать или перерабатывать остатки от раскроев заготовок.

6. Необходимо более тонкое понимание поведения показателя прибыли.

Так как проблема раскроя на практике является трехмерной, то в будущих работах необходимо стремиться к учету этого фактора.

\section{ЗАКЛЮЧЕНИЕ}

В работе рассматривается экономико-математическая модель, позволяющая строить устойчивые цепочки поставок сырья, и отличающаяся возможностью учитывать следующие факторы лесопромышленного производства: нормы затрат сырья при производстве всех товаров, неприкосновенный и максимальный запас сырья на складе, время заявки с биржи в пути, объем спроса на рынке товаров из разных типов сырья, зависимость цен от объема спроса во времени, а также отличающаяся комплексной оптимизацией четырех наиболее важных процессов производства: раскрой плит, выпуск готовой продукции, формирование цепочек поставок сырья и транспортировкой готовых видов продукции до покупателя. Целевая функция модели сформулирована с учетом принципа максимизации конечного показателя прибыли. Выбран стандартный алгоритм по поиску решения. Теоретическая значимость исследо- 
Влияние подзадачи о раскрое при оценке иелесообразности проведения закупок лесного сырья ...

вания заключается в разработке новой модели по оценке целесообразности проведения закупок сырья с биржи с учетом возможности раскроя и формированием плана транспортировки готовой продукции до покупателей.

Недостатками работы можно назвать:

1. Отсутствие учета некоторых часто возникающих рисков, связанных с отказом от сырья.

2. Отсутствие учета рисков, связанных с транспортной системой дорог.

3. Не рассматривает возможность получения в аренду делян.

4. Не рассматривается возможность при отрицательном значении прибыли в какой-либо день получения инвестиций.

5. Отсутствие технологического учета остатков после раскроя.

6. Необходим более тонкий анализ показателя прибыли во времени.

7. В работе рассмотрен одномерный раскрой, однако, в жизни встречается зачастую трехмерный раскрой.

Перечислим преимущества предложенной модели:

1. Количество ограничений оптимизировано до максимума.

2. Линейная модель.

3. Существует возможность ускорения поиска решения задачи при использовании параллельного программирования.

4. Учет четырех наиболее важных процессов лесопромышленного предприятия: задача раскроя, проблема определения оптимального объема производства, транспортная задача и устойчивое формирование цепочек поставок сырья с биржи.

5. При достаточно больших выборках стандартный алгоритм поиска решения способен за незначительный срок определять решения для рассматриваемой комплексной и актуальной задачи.

Авторская модель имеет большое множество для дальнейшей модернизации путем включения различных дополнительных экономико-технологических процессов.

Модель прошла апробацию с использованием официальных данных нескольких предприятий и Санкт-Петербургской Международной
Товарно-сырьевой Биржи. Поведение прибыли показывает, что в примерно 95 \% случаев существенную эффективность взаимодействия с товарно-сырьевой биржей, что подтверждает искомую гипотезу исследования.

\section{БЛАГОДРАНОСТИ}

Работа была подготовлена при поддержке DAAD (German Academic Exchange Service) и Министерства Науки и Высшего образования Российской Федерации в рамках программы «Иммануил Кант».

\section{КОНФЛИКТ ИНТЕРЕСОВ}

Автор декларирует отсутствие явных и потенциальных конфликтов интересов, связанных с публикацией настоящей статьи.

\section{СПИСОК ЛИТЕРАТУРЫ}

1. Kondili E., Pantelides C. C., Sargent R. W. H. A General Algorithm for Short-term Scheduling of Batch Operations-I. MILP Formulation // Computers \& Chemical Engineering. - 1993. Vol. 17. - P. 211-227. DOI: 10.1016/00981354(93)80015-F

2. Georgiadis G. P., Elekidis A. P., Georgiadis M. C. Optimization-Based Scheduling for the Process Industries: From Theory to Real-Life Industrial Applications // Processes. 2019. - Vol. 7. - P. 438. https://doi.org/10.3390/ pr7070438

3. Bixby R., Rothberg E. Progress in Computational Mixed Integer Programming - A Look Back from the Other Side of the Tipping Point // Annals of Operations Research. - 2007. Vol. 149. - P. 37-41. DOI: 10.1007/s10479-0060091-y

4. Shah N., Pantelides C. C., Sargent R. W. H. A General Algorithm for Short-Term Scheduling of Batch-Operations 2. Computational Issues // Computers \& Chemical Engineering. - 1993. Vol. 17. - P. 229-244. DOI: 10.1016/00981354(93)80016-G

5. Lee H., Maravelias C. T. Combining the Advantages of Discrete- and Continuous-Time Scheduling Models: Part 1. Framework and Math- 


\section{Р. С. Рогулин}

ematical Formulations // Computers \& Chemical Engineering. - 2018. - Vol. 116. - P. 176-190. DOI: 10.1016/j.compchemeng.2017.12.003

6. Castro P. M., Mostafaei H. Batch-centric Scheduling Formulation for Treelike Pipeline Systems with Forbidden Product Sequences // Computers \& Chemical Engineering. - 2018. Vol. 122. - P. 2-18. DOI: 10.1016/j.compchemeng.2018.04.027

7. Georgiadis G. P., Ziogou C., Kopanos G. M., Garcia M., Cabo D., Lopez M., Georgiadis M. C. Production Scheduling of Multi-Stage, Multi-product Food Process Industries. In Proceedings of the 28th European Symposium on Computer Aided Process Engineering, Graz, Austria, 10-13 June 2018. - P. 1075-1080. DOI: 10.1016/ B978-0-444-64235-6.50188-1

8. Georgiadis G. P., Kopanos G. M., Karkaris A., Ksafopoulos H., Georgiadis M. C. Optimal Production Scheduling in the Dairy Industries // Industrial \& Engineering Chemistry Research. 2019. - Vol. 58. - P. 6537-6550. DOI: 10.1021/ acs.iecr.8b05710

9. Elekidis A., Corominas F., Georgiadis M. C. Optimal short-term Scheduling of Industrial Packing Facilities. In Proceedings of the 29th European Symposium on Computer Aided Process Engineering, Eindhoven, The Netherlands, 1619 June 2019. DOI: 10.1016/B978-0-12-8186343.50198-3

10. Рогулин Р. С., Мазелис Л. С. Алгоритм и математическая модель формирования устойчивых цепочек поставок древесного сырья из регионов России: сравнение и анализ // Вестник Пермского университета. Сер. «Экономика». - 2020. - Т. 15, № 3. - С. 385-404. DOI: 10.17072/1994-9960-2020-3-385-404

11. Georgios P. G., Pampin B. M., Cabo D. A., Georgiadis M. C. Optimal production scheduling of food process industries // Computers \& Chemical Engineering. - 2020. - Vol. 134. DOI: 10.1016/j.compchemeng.2019.106682.

12. Zhiru L., Wei X., Huibin Sh., Qingshan Zh., Fengyi H. Multiobjective Optimization Model of Production Planning in Cloud Manufacturing Based on TOPSIS Method with Combined Weights // Complexity. - 2019. - Vol. 2019. DOI: 10.1155/2019/7503176
13. Zhiyi Zh., Shuhong Ch., Ka Y. Ch. A New Model of Manufacturer's Optimal Product Supply Strategy in the Context of Precision Marketing: Based on Real Demand Pattern // Mathematical Problems in Engineering. - 2020. - Vol. 2020. DOI: $10.1155 / 2020 / 6031563$

14. Zhuo Z. Y., Chen S. H., Yan H., He Y. Models for retailers' supply strategies aimed at individual customers under the real demand pattern // Mathematical Problems in Engineering. - 2020. Vol. 1. - P. 11. DOI: 10.1155/2020/6031563

15. Zhuo Z., Chau K. Y., Huang S. Z., Ip Y. K. Mathematical modelling of optimal product supply strategies for manufacturer-to-group customers based on semi-real demand patterns // International Journal of Operational Research. - 2020. - Vol. 1, No. 1. - P. 1-8. DOI: $10.1177 / 1847979020941489$

16. Zhuo Z. New mathematical model of retailer-to-individual customer optimal product supply strategies under false demand pattern: customer discount mode // Journal of Mathematics Research. - 2020. - Vol. 12, No. 1. - P. 36-42. DOI: 10.5539/jmr.v12n1p36

17. Zhuo Z. Y. Research on optimal product supply strategies for manufacturer-to-group customer under a real demand pattern // International Journal of Operational Research. - 2020. Vol. 1. - P. 36. DOI: 10.5539/jmr.v12n1p36

18. Ouyang W., Giancarlo D. A., Iiro H., Ala B., Schneider St. M., Roth M., Imsland L. Optimal production and maintenance scheduling for a multiproduct batch plant considering degradation // Computers \& Chemical Engineering. 2020. - Vol. 135. - P. 34-39. DOI:10.1016/j. compchemeng.2020.106734.

19. Rezig S., Ezzeddine W., Turki S., Rezg N. Mathematical Model for Production Plan Optimization - A Case Study of Discrete Event Systems // Mathematics. - 2020. - Vol. 8. - P. 955. DOI:10.3390/math8060955

20. Рогулин Р. С. Модель оптимизации плана закупок сырья из регионов России лесоперерабатывающим комплексом // Бизнес-информатика. - 2020. - Т. 14, № 4. - С. 19-35. DOI: 10.17323/2587-814X.2020.4.19.35

21. Рогулин Р. С. Моделирование перспектив взаимодействия предприятия лесопромышленного комплекса и товарно-сырьевой 
Влияние подзадачи о раскрое при оценке иелесообразности проведения закупок лесного сырья ...

биржи России // Journal of Applied Economic Research. - 2020. - T. 19, № 4. - C. 489-511. DOI: 10.15826/vestnik.2020.19.4.023.

22. Khan R., Pruncu C. I., Khan A. S., Naeem K., Abas M., Khalid Q. S., Aziz A. A Mathematical Model for Reduction of Trim Loss in Cutting Reels at a Make-to-Order Paper Mill // Applied Sciences. - 2020. - Vol. 10. - P. 52-74. DOI:10.3390/app10155274

23. Song Ch., Gong K., Bu J., Huang L. Research on Stability of Optimal Sheet-cutting Strategy Based on Improved Real-Coded Genetic Algorithm. E3S Web of Conferences. 2020. - Vol. 162. - P. 3-7. DOI: 10.1051/e3sconf/202016203007.

Рогулин Родион Сергеевич - ассистент кафедры математики и моделирования Владивостокского государственного университета

E-mail: rafassiaofusa@mail.ru

ORCID iD: https://orcid.org/ORCID: 0000-0002-3235-6429
24. Song K., Gong J., Bu L. Research on Optimal Sheet-cutting Strategy Based on Improved Genetic Algorithm // E3S Web Conferences. - Vol. 162. - P. 30-37. DOI: 10.1051/e3sconf $/ 202016204107$

25. Petunin A. A., PolishchukE. G., Ukolov S. S. On the new Algorithm for Solving Continuous Cutting Problem // IFAC-Papers OnLine. 2019. - Vol. 52. - P. 2320-2325. DOI: 10.1016/j. ifacol.2019.11.552

Received 05.04.2021

Accepted 19.07.2021

\title{
THE CUTTING STOCK PROBLEM IN RELATION TO THE ASSESSMENT OF THE FEASIBILITY OF PURCHASING TIMBER FROM THE COMMODITY MARKET
}

\author{
(C) 2021 R. S. Rogulin \\ Vladivostok State University of Economics and Service \\ 41, Gogolya Street, 690014 Vladivostok, Russian Federation
}

\begin{abstract}
Annotation. The article considers a mathematical model that can be used to determine the optimal plan for the procurement of raw materials according to the preset timing of applications, determine the production plan, and calculate the optimal plan for the delivery of products. The specific feature of the model is that it can also plan the cutting of OSBs into workpieces of various lengths. It also considers the dependency between the price and the level of demand for finished goods. The purpose of our research was to assess the economic feasibility of interaction between a timber industry enterprise (with different levels of price and demand) and an international commodity market. Our hypothesis is that the interaction between the timber industry and the commodity market may be beneficial for the enterprise. The relevance of the study is determined by the lack of studies on the interaction of timber industry enterprises with the commodity market, with the latter being a source of raw materials. Various modelling methods were used for the assessment: mixed-integer linear programming (drawing up a model that finds the optimal vectors for cutting, procurement of raw materials, production of goods and their transportation based on the graph), the Monte Carlo method (generating initial conditions and parameters),

Rogulin Rodion S. e-mail: rafassiaofusa@mail.ru and simulation modelling (for each state the corresponding task was solved). The main drawback
\end{abstract}




\section{Р. С. Рогулин}

of the model is that it requires a significant amount of RAM. The practical significance of the study is to prove the economic feasibility of purchasing raw materials from the commodity market of Russia. The theoretical value of the study is that it develops a new model for assessing the feasibility of purchasing raw materials using the market mechanisms. Scientific novelty is determined by the fact that it suggests a mathematical model for the formation of supply chains, drawing up a cutting plan and production volumes, taking into account the demand and available volume of raw materials. The model was tested using the data of one of the enterprises of the timber industry of the Primorsky Krai. It was used to optimize the volume of manufactured products and purchased raw materials from each region, as well as the number of cuts for each OSB and the stock of raw materials at the enterprise's warehouse. Based on the results of testing, we assessed the feasibility of cooperation between the company and the commodity market. In $95 \%$ of situations the cooperation is highly beneficial.

Keywords: cutting problem, supply chain, raw materials, timber industry, optimal plan, production, mathematical modelling.

\section{CONFLICT OF INTEREST}

The author declare the absence of obvious and potential conflicts of interest related to the publication of this article.

\section{REFERENCES}

1. Kondili E., Pantelides C. C., Sargent R. W. H. (1993). A General Algorithm for Short-term Scheduling of Batch Operations-I. MILP Formulation. Computers \& Chemical Engineering. Vol. 17. P. 211-227. DOI: 10.1016/00981354(93)80015-F

2. Georgiadis G. P., Elekidis A. P., Georgiadis M. C. (2019). Optimization-Based Scheduling for the Process Industries: From Theory to Real-Life Industrial Applications. Processes. Vol. 7, P. 438. https://doi.org/10.3390/pr7070438

3. Bixby R., Rothberg E. (2007). Progress in Computational Mixed Integer Programming A Look Back from the Other Side of the Tipping Point. Annals of Operations Research. Vol. 149. P. 37-41. DOI: 10.1007/s10479-006-0091-y

4. Shah N., Pantelides C. C., Sargent R. W. H. (1993). A General Algorithm for Short-Term Scheduling of Batch-Operations 2. Computational Issues. Computers \& Chemical Engineering, Vol. 17. P. 229-244. DOI: 10.1016/00981354(93)80016-G

5. Lee H., Maravelias C. T. (2018). Combining the Advantages of Discrete- and Continuous-Time Scheduling Models: Part 1. Framework and Mathematical Formulations. Computers \& Chemical Engineering. Vol. 116. P. 176-190. DOI: 10.1016/j.compchemeng.2017.12.003
6. Castro P. M., Mostafaei H. (2018). Batch-centric Scheduling Formulation for Treelike Pipeline Systems with Forbidden Product Sequences. Computers \& Chemical Engineering. Vol. 122. P. 2-18. DOI: 10.1016/j.compchemeng.2018.04.027

7. Georgiadis G. P., Ziogou C., Kopanos G. M., Garcia M., Cabo D., Lopez M., Georgiadis M. C. (2018). Production Scheduling of Multi-Stage, Multi-product Food Process Industries. In Proceedings of the 28th European Symposium on Computer Aided Process Engineering, Graz, Austria, 10-13 June 2018; P. 1075-1080. DOI: 10.1016/B978-0-444-64235-6.50188-1

8. Georgiadis G. P., Kopanos G. M., Karkaris A., Ksafopoulos H., Georgiadis M. C. (2019). Optimal Production Scheduling in the Dairy Industries. Industrial \& Engineering Chemistry Research. Vol. 58. P. 6537-6550. DOI: 10.1021/acs. iecr.8b05710

9. Elekidis A., Corominas F., Georgiadis M. C. (2019). Optimal short-term Scheduling of Industrial Packing Facilities. In Proceedings of the 29th European Symposium on Computer Aided Process Engineering, Eindhoven, The Netherlands, 16-19 June 2019. DOI: 10.1016/B978-012-818634-3.50198-3

10. Rogulin R. S., Mazelis L. S. (2020). Algoritm i matematicheskaya model' formirovaniya ustoychivykh tsepochek postavok drevesnogo syr'ya iz regionov Rossii: sravneniye i analiz (Algorithm and mathematical model for the formation of sustainable supply chains of wood raw materials from the regions of Russia: comparison and analysis). Bulletin of Perm University. Ser. "Economy". Vol. 15, No 3. P. 385-404. DOI: 10.17072/1994-9960-2020-3-385-404 (in Russ.) 
11. Georgios P. G., Pampín B. M., Cabo D. A., Georgiadis M. C. (2020). Optimal production scheduling of food process industries. Computers \& Chemical Engineering. Vol. 134. DOI: 10.1016/j.compchemeng.2019.106682.

12. Zhiru L., Wei X., Huibin Sh., Qingshan Zh., Fengyi H. (2019). Multiobjective Optimization Model of Production Planning in Cloud Manufacturing Based on TOPSIS Method with Combined Weights. Complexity. Vol. 2019. DOI: 10.1155/2019/7503176

13. Zhiyi Zh., Shuhong Ch., Ka Y. Ch. (2020). A New Model of Manufacturer's Optimal Product Supply Strategy in the Context of Precision Marketing: Based on Real Demand Pattern. Mathematical Problems in Engineering, Vol. 2020. DOI: $10.1155 / 2020 / 6031563$

14. Zhuo Z. Y., Chen S. H., Yan H., He Y. (2020). Models for retailers' supply strategies aimed at individual customers under the real demand pattern. Mathematical Problems in Engineering. Vol. 1. P. 11. DOI: 10.1155/2020/6031563

15. Zhuo Z., Chau K. Y., Huang S. Z., Ip Y. K. (2020). Mathematical modelling of optimal product supply strategies for manufacturer-to-group customers based on semi-real demand patterns. International Journal of Operational Research. Vol. 1, No. 1. P. 1-8. DOI: 10.1177/1847979020941489

16. Zhuo Z. (2020). New mathematical model of retailer-to-individual customer optimal product supply strategies under false demand pattern: customer discount mode. Journal of Mathematics Research. Vol. 12, No. 1. P. 36-42. DOI: 10.5539/ jmr.v12n1p36

17. Zhuo Z. Y. (2020). Research on optimal product supply strategies for manufacturer-to-group customer under a real demand pattern. International Journal of Operational Research. Vol. 1. P. 36. DOI: 10.5539/jmr.v12n1p36

18. Ouyang W., Giancarlo D. A., Iiro H., Ala B., Schneider St. M., Roth M., Imsland L. (2020). Optimal production and maintenance schedul- ing for a multiproduct batch plant considering degradation. Computers \& Chemical Engineering, Vol. 135. P. 34-39. DOI:10.1016/j.compchemeng.2020.106734.

19. Rezig S., Ezzeddine W., Turki S., Rezg N. (2020). Mathematical Model for Production Plan Optimization - A Case Study of Discrete Event Systems. Mathematics. Vol. 8. P. 955. DOI:10.3390/math8060955

20. Rogulin R. S. (2020). A model for optimizing plans for procurement of raw materials from regions of Russia in a timber-processing enterprise. Business Informatics. No 4. P. 19-35. DOI: 10.17323/2587-814X.2020.4.19.35

21. Rogulin R. S. (2020) Modeling of Promising Interaction Between a Timber Industry Enterprise and a Commodity Exchange in Russia. Journal of Applied Economic Research. Vol. 19. No. 4. P. 489-511. DOI: $10.15826 /$ vestnik.2020.19.4.023. (in Russ.)

22. Khan R., Pruncu C. I., Khan A. S., Naeem K., Abas M., Khalid Q. S., Aziz A. (2020). A Mathematical Model for Reduction of Trim Loss in Cutting Reels at a Make-to-Order Paper Mill. Applied Sciences. Vol. 10. P. 52-74. DOI:10.3390/ app10155274

23. Song Ch., Gong K., Bu J., Huang L. (2020). Research on Stability of Optimal Sheet-cutting Strategy Based on Improved Real-Coded Genetic Algorithm. E3S Web of Conferences. Vol. 162. P. 3-7. DOI: 10.1051/e3sconf/202016203007.

24. Song K., Gong J., Bu L. (2020). Research on Optimal Sheet-cutting Strategy Based on Improved Genetic Algorithm. E3S Web Conferences. Vol. 162. P. 30-37. DOI: 10.1051/e3sconf/202016204107

25. Petunin A. A., PolishchukE. G., Ukolov S. S. (2019). On the new Algorithm for Solving Continuous Cutting Problem. IFAC-Papers OnLine. Vol. 52. P. 2320-2325. DOI: 10.1016/j.ifacol.2019.11.552

Rogulin Rodion S. - lecturer, Department of Mathematics and Modelling, Vladivostok State University of Economics and Service.

E-mail: rafassiaofusa@mail.ru

ORCID iD: https://orcid.org/0000-0002-3235-6429 